Marquette University

e-Publications@Marquette

Mechanical Engineering Faculty Research and

Publications

Mechanical Engineering, Department of

5-11-2002

\title{
Sufficient Conditions Used in Admittance Selection for Planar Force-guided Assembly
}

Shuguang Huang

Marquette University, shuguang.huang@marquette.edu

Joseph M. Schimmels

Marquette University, joseph.schimmels@marquette.edu

Accepted version. Published as a part of the Proceedings. ICRA '02. IEEE International Conference on Robotics and Automation, 2002. DOI. (C) 2002 IEEE. Used with permission. 


\section{Marquette University \\ e-Publications@Marquette}

\section{Mechanical Engineering Faculty Research and Publications/College of Engineering}

This paper is NOT THE PUBLISHED VERSION; but the author's final, peer-reviewed manuscript. The published version may be accessed by following the link in the citation below.

IEEE Transactions on Robotics and Automation, Vol. 1 (2002): 538-543. DOI. This article is (c) Institute of Electrical and Electronics Engineers and permission has been granted for this version to appear in e-Publications@Marquette. Institute of Electrical and Electronics Engineers does not grant permission for this article to be further copied/distributed or hosted elsewhere without the express permission from Institute of Electrical and Electronics Engineers.

\section{Sufficient Conditions Used in Admittance Selection for Planar Force-Guided Assembly}

Shuguang Huang

Dept. of Mech. \& Ind. Eng., Marquette University, Milwaukee, WI

J.M. Schimmels

Dept. of Mech. \& Ind. Eng., Marquette University, Milwaukee, WI 


\section{SECTION I.}

\section{Introduction}

ADMMITTANCE control has been used in assembly tasks to provide force regulation and guidance. In this application, admittance is a mapping of contact forces to velocity changes for the held body. To achieve reliable assembly, an appropriate admittance must be selected. For a linear admittance behavior, the spatial control law has the form:

$$
\mathbf{v}=\mathbf{v}_{0}+\mathbf{A f}(1)
$$

where $V_{o}$ is the nominal velocity (a 6-vector), $f$ is the contact force (wrench) measured in the body frame (a 6-vector), and $A$ is the admittance matrix (a $6 \times 6$ matrix).

Many researchers have addressed the use of admittance for force-guidance. Whitney [1], [2] proposed that the compliance of a manipulator be structured so that contact forces lead to decreasing errors. Peshkin ${ }^{[3]}$ addressed the synthesis of an accommodation (inverse damping) matrix by specifying the desired force/motion relation at a sampled set of positional errors for a planar assembly task. An unconstrained optimization was then used to obtain an accommodation matrix. Asada [4] used a similar optimization procedure for the design of an accommodation neural network rather than an accommodation matrix. Others ${ }^{[5]}$, [6] provided synthesis procedures based on spatial intuitive reasoning. None of these approaches, however, ensures that the admittance selected will, in fact, be reliable.

A reliable admittance selection approach is to design the control law so that, at each possible part misalign-ment, the contact force always leads to a motion that reduces the existing misalignment. The approach is referred to as force-assembly [7], [8], [9]. A condition for forceassembly is that the admittance matrix $A$ must be positive semidefinite ${ }^{[7]}$.

For force-assembly, the motion resulting from contact must reduce misalignment. Since the configuration space of rigid body is non-Euclidian, there is no natural metric for finite spatial error. In [10], a rigid body metric is established. This metric is based on the Euclidean distance between one specific point in the body and its location when properly positioned. However, as the choice of the point is configuration dependent, the calculation of the metric for an arbitrary configuration is difficult.

In this paper, we consider a measure of error based on the Euclidean distance between a single (fixed) point on the held body and its location when properly positioned. The error reduction condition of force-assembly requires that, at each possible misalignment, the contact force yields a motion that reduces this distance. This condition can be expressed mathematically if we let $d$ (a 6-vector for spatial motion) be the line vector from the point at its proper mated position to its current position. Then, for error reducing motion, the condition is:

$$
\mathbf{d}^{T} \mathbf{v}=\mathbf{d}^{T}\left(\mathbf{v}_{0}+\mathbf{A f}\right)<0 \text { (2) }
$$


which must be satisfied for all possible misalignments. Because the line vector $d$ depends on the rigid body configuration and because the number of configurations is infinite, it is impossible to test the error-reducing motion for all misalignments, In application, the misalignments of the rigid body are bounded by the accuracy of the robotic manipulator. Those misalignments on the "boundary" are of interest. The contribution of this paper is to show that, by satisfying the error-reduction conditions at a finite number of configurations, the error reduction requirement is also ensured for all configurations within the bounded area.

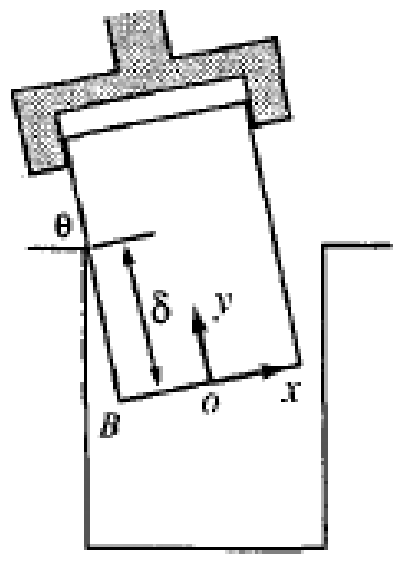

(a)

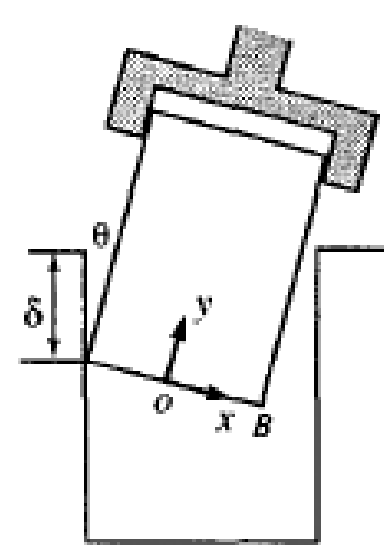

(b)

Fig. 1. Planar single-point contact. (a) edge-vertex contact state: an edge of the held body in contact with a vertex of the mating fixtured part. (b) vertex-edge contact state: a vertex of the held body in contact with an edge of the mating fixtured part.

This paper considers rigid body assembly with the following restrictions: planar motion, frictionless con-tact, and single-point contact.

Planar bodies in single-point contact have two types of contact states. One is referred to as "edge-vertex" contact; the other is referred to as "vertex-edge" contact. In "edge-vertex" contact, one edge of the held body is in contact with one vertex of the mating fixtured part (Fig. 1a). In "vertex-edge" contact, one vertex of the held body is in contact with one edge of its mating part (Fig. 1b).

In this paper, sufficient conditions for error reduction for edge-vertex and vertex-edge contact states are derived in Section 2 and Section 3, respectively. These conditions show that an admittance matrix satisfying the error reduction conditions at the boundaries of a set of contact configurations, also satisfies the error-reduction conditions at all intermediate config-urations. A brief discussion and a summary are presented in Sections 4 and 5. 


\section{SECTION II.}

\section{Edge-Vertex Contact State}

In this section, "edge-vertex" contact is considered. As shown in Fig. 1a, one edge of the held body is in contact with a vertex of the mating part.

Let $B$ be a point on the body, $\theta$ be the angle of rotation of the body, and $\delta$ be the distance of the contact normal to the body coordinate frame. Then for a given contact state, the configuration of the body can be determined by two parameters, $(\delta, \theta)$

Suppose that $\theta$ varies within the range of $[\theta m, \theta M]$, and $\delta$ varies within the range of $[0, \Delta]$. We prove that, if an admittance matrix A satisfies a set of conditions at the "boundary" points $(\theta=$ $\theta_{m}, \theta_{M}$, and $\left.\delta=0, \Delta\right)$ then the A matrix ensures the error-reducing motion of $B$ for configurations $\theta \in\left[\theta_{m}, \theta_{M}\right], \delta \in[0, \Delta]$. To prove the results, we first consider orientational and translational variation separately, Finally, the general edge-vertex case is considered.

\section{A. Orientational Variation}

First we consider only variation of orientation as illustrated in Fig. 2a. In this case, only rotation about the contact point is allowed, We prove that, if for $\theta \mathrm{m}$ and $\theta M$, the system satisfies the error reduction conditions, then for any $\theta \in\left[\theta_{m}, \theta_{M}\right]$ the system will also satisfy the error reduction conditions.

Let $B 1$ and $B 2$ be the position of $B$ at $\theta m$ and $\theta M$, and $\mathbf{d} 1$ and $\mathbf{d} 2$ be the line vectors from the home position $B h$ to $B 1$ and $B 2$, respectively.

Suppose that a.t $\theta m$ and $\theta M$, the motion is error reducing for point $B$. Then, at each of the two con-figurations, the distance between $B$ and $B h$ must be reduced by the motion. By Eq. (2), we have:

$$
\begin{gathered}
\mathbf{d}_{1}{ }^{T} \mathbf{v}_{0}+\mathbf{d}_{1}{ }^{T} \mathbf{A} \mathbf{w}_{1} \leq 0, \\
\mathbf{d}_{2}{ }^{T} \mathbf{v}_{0}+\mathbf{d}_{2}{ }^{T} \mathbf{A} \mathbf{w}_{2} \leq 0
\end{gathered}
$$

where $\boldsymbol{v} 0$ is the nominal velocity, $\mathrm{A}$ is the admittance matrix, $\mathbf{w} 1$ and $\mathbf{w} 2$ are the wrenches corresponding to $\theta \mathrm{m}$ and $\theta M$, respectively. Any positive combination of Eq. (3) and (4) will also be satisfied if (3) and (4) are independently satisfied, i.e., for $\alpha, \beta \geq 0$,

$$
\left(\alpha \mathbf{d}_{1}+\beta \mathbf{d}_{2}\right)^{T} \mathbf{v}_{0}+\alpha \mathbf{d}_{1}{ }^{T} \mathbf{A} \mathbf{w}_{1}+\beta \mathbf{d}_{2}{ }^{T} \mathbf{A} \mathbf{w}_{2} \leq 0 . \text { (5) }
$$

Since the contact point is constant, for any $\theta \in\left[\theta_{m}, \theta_{M}\right]$, the point $B$ must be on the arc connecting $B 1$ and $B 2$. Thus, there exist two non-negative numbers $\alpha \leq 1$ and $\beta \leq 1$, such that the position vector of $B, \mathbf{d}$, can be expressed for any intermediate configuration as: 


$$
\mathbf{d}=\alpha \mathbf{d}_{1}+\beta \mathbf{d}_{2 \cdot(6)}
$$

Since in the body frame

$$
\mathbf{w}_{1}=\mathbf{w}_{2}=\mathbf{w}
$$

thus

$$
\mathbf{d}^{T} \mathbf{v}_{0}+\mathbf{d}^{T} \mathbf{A w} \leq 0
$$

This means that if Eqs. (3) and (4) are satisfied, the error reduction condition is satisfied for any $\theta \in[\theta m, \theta M]$.

\section{B. Translational Variation}

Next we consider only the variation of translation as illustrated in Fig. 2b. Let $\Delta$ be the distance between the two extremal configurations shown in Fig. 2b. We prove that, if at the two ends of the translation (cor-responding to $\delta=0$ and $\delta=\Delta$ ) the system yields an error reducing motion and the A matrix satisfies, some additional conditions, then for any configuration between $\delta \in$ $[0, \Delta]$, the system also yields an error-reducing motion.

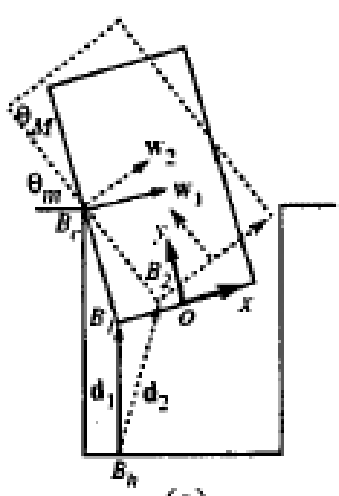

(a)

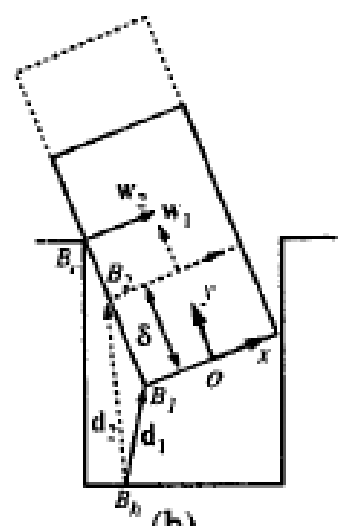

(b)

Fig. 2. Edge-vertex contact state. (a) orientational variation. (b) translational variation.

\section{B.1 Determining the Contact Force}

Unlike the orientational case, the contact wrench changes in the body coordinate as the contact point changes (although its direction is constant). Thus, the contact force must be determined for different contact configurations. 
Since friction is not considered, the force is along the normal of the body surface. In the coordinate frame shown in Fig. $2 \mathrm{~b}$, the wrench obtained at an arbitrary configuration $\delta$ between the two extremals can be expressed as:

$$
\mathbf{w}_{\delta}=\left[f_{x}, 0,-f_{x} \delta\right]^{T}, \delta \in[0, \Delta] .(8)
$$

Because the contact point cannot penetrate into either body, the reciprocal condition must be satisfied:

$$
\mathbf{w}_{n}^{T} \mathbf{v}_{0}+\mathbf{w}_{n}^{\mathbf{T}} \mathbf{A} \mathbf{w}_{\delta}=0 \text { (9) }
$$

where $\boldsymbol{w} n$ is the unit wrench corresponding to the surface normal and has the form:

$$
\mathbf{w}_{n}=[1,0,-\delta]^{T} \text {. }
$$

Since the contact is frictionless, the contact wrench $\boldsymbol{w}$ must have the same direction of $\boldsymbol{w} n$. Thus,

$$
\mathbf{w}_{\delta}=\mathbf{w}_{n} \phi(11)
$$

where $\phi \geq 0$ is the magnitude of the contact force.

Suppose that the nominal velocity only has translational components. Then $v 0$ has the form:

$$
\mathbf{v}_{0}=\left[v_{x}, v_{y}, 0\right]^{T}
$$

The magnitude $\phi$ can be determined by substituting Eq. (11) into Eq. (9) yielding

$$
\phi=-\frac{\mathbf{w}_{n}^{T} \mathbf{v}_{0}}{\mathbf{w}_{n}^{T} \mathbf{A} \mathbf{w}_{n}}=-\frac{v_{x}}{a_{11}-2 a_{13} \delta+a_{33} \delta^{2}}
$$

where $a_{i j}$ is the $(i, j)^{t h}$ entry of $\mathrm{A}$ and $v x$ is the $x$ - component of $v 0$. Since A is positive semidefinite ${ }^{[7]}$, it can be proved that $a_{11}-2 a_{13} \delta+a_{33} \delta^{2} \geq 0$ for all $\delta \in[0, \Delta]$.

\section{B.2 Error Reduction Conditions}

Suppose that for $\delta=0$ and $\delta=\Delta$ the error reduction conditions are satisfied, i.e.,

$$
\begin{aligned}
& \mathbf{d}_{1}{ }^{T} \mathbf{v}_{0}+\mathbf{d}_{1}{ }^{T} \mathbf{A} \mathbf{w}_{1} \leq 0 \\
& \mathbf{d}_{2}{ }^{T} \mathbf{v}_{0}+\mathbf{d}_{2}{ }^{T} \mathbf{A} \mathbf{w}_{2} \leq 0
\end{aligned}
$$

For any intermediate configuration, since the point $B t$ must be on the line segment $B 1 B 2$, the line vector $\boldsymbol{d} \delta$ associated with $B \delta$ can be expressed as: 


$$
\mathbf{d}_{\delta}=\mathbf{d}_{1}+\mathbf{d}_{0} \delta, \delta \in[0, \Delta](15)
$$

where $\boldsymbol{d} 0$ is defined as:

$$
\mathbf{d}_{0}=\frac{\mathbf{d}_{2}-\mathbf{d}_{1}}{\left\|\mathbf{d}_{1}^{\prime}-\mathbf{d}_{2}^{\prime}\right\|}
$$

and $\mathbf{d}_{i}^{\prime}(i=1,2)$ is the first two components (the position 2-vector) of $\boldsymbol{d} i$.

Thus, for any intermediate configuration, the error reduction condition is a function of $\delta$ :

$$
\begin{aligned}
& E(\delta)=\mathbf{d}_{\delta}^{T} \mathbf{v}_{0}+\mathbf{d}_{\delta^{T}} \mathbf{A} \mathbf{w}_{\delta} \\
& \quad=\left(\mathbf{d}_{1}+\delta \mathbf{d}_{0}\right)^{T} \mathbf{v}_{0}+\left(\mathbf{d}_{1}+\delta \mathbf{d}_{0}\right)^{T}\left(\mathbf{a}_{1}-\mathbf{a}_{3} \delta\right) \phi
\end{aligned}
$$

where $\phi$ is the magnitude of the contact force and $\boldsymbol{a} i$ is the $i$ th column of A.

Substituting Eq. (12) into Eq. (17), we have:

$$
E(\delta)=\left(\mathbf{d}_{1}+\delta \mathbf{d}_{0}\right)^{T} \mathbf{v}_{0}-\frac{\left(\mathbf{d}_{1}+\delta \mathbf{d}_{0}\right)^{T}\left(\mathbf{a}_{1}-\mathbf{a}_{3} \delta\right) v_{x}}{a_{11}-2 a_{13} \delta+a_{33} \delta^{2}}
$$

View Source

Since $a_{11}-2 a_{13} \delta+a_{33} \delta^{2}>0$ and only the sign of $E(\delta)$ is considered, the error reduction condition can be equivalently written as a polynomial of $\delta$ :

$$
E(\delta)=\left(\mathbf{d}_{1}+\delta \mathbf{d}_{0}\right)^{T} \mathbf{v}_{0}-\frac{\left(\mathbf{d}_{1}+\delta \mathbf{d}_{0}\right)^{T}\left(\mathbf{a}_{1}-\mathbf{a}_{3} \delta\right) v_{x}}{a_{11}-2 a_{13} \delta+a_{33} \delta^{2}}
$$

It can be seen that $F(\delta)$ is a third order polynomial of $\delta$ with $F(0)<0$ and $F(\Delta)<0$. To ensure $F(\delta) \leq 0$ for all $\delta \in[0, \Delta], F(\delta)$ must have no real root in $[0, \Delta]$.

Consider the two sequences S1 and S2 defined by

$$
\begin{aligned}
& S_{1}: F(0), F^{\prime}(0), F^{(2)}(0), F^{(3)}(0) ; \\
& S_{2}: F(\Delta), F^{\prime}(\Delta), F^{(2)}(\Delta), F^{(3)}(\Delta) .
\end{aligned}
$$

By the Fourier Theorem, $F$ has no real root in $[0, \Delta]$ if and only if the numbers of sign changes for $S 1$ and $S 2$ are the same. Since the coefficients of the poly-nomial depend on the the admittance matrix A, each element of $S 1$ and $S 2$ is a function of $A$. Therefore, the error reduction conditions on the admittance matrix can be obtained. This condition can be stated as the following: 


\section{Condition A}

The admittance matrix A satisfies the condition that the sequences $S 1 \mathrm{in} \mathrm{Eq}, \underline{(18)}$ and $S 2$ in Eq, (19) have the same number of sign changes.

Thus, if the admittance matrix A satisfies Condition A and yields motions of error reduction at the two boundary configurations, then the system yields a motion of error reduction for any intermediate configuration.

It is noted that it is not difficult to satisfy Condition A. Since $F(\delta)$ is a polynomial of third order, it can be proved that if both $F(0)$ and $F(\Delta)$ are negative and $F^{\prime}(0)<0, F^{\prime}(\Delta)>0$, then Condition $A$ is satisfied for the interval $[0, \Delta]$. Thus, Condition $A$ can be expressed in terms of $A$ as:

$$
\begin{gathered}
F^{\prime}(0)=\mathbf{d}_{1}^{T} \mathbf{v}_{0} a_{33}-2 a_{13} \mathbf{d}_{0}^{T} \mathbf{v}_{0}+\mathbf{d}_{0}^{T} \mathbf{a}_{3} v_{x}<0, \\
F^{\prime}(\Delta)=3 a_{33} \mathbf{d}_{0}^{T} \mathbf{v}_{0} \Delta^{2}+2\left(\mathbf{d}_{1}^{T} \mathbf{v}_{0} a_{33}-2 a_{13} \mathbf{d}_{0}^{T} \mathbf{v}+\right. \\
\left.\mathbf{d}_{0}^{T} \mathbf{a}_{3} v_{x}\right) \Delta+\left(a_{11} \mathbf{d}_{0}^{T} \mathbf{v}_{0}-2 a_{13} \mathbf{d}_{1}^{T} \mathbf{v}_{0}+\right. \\
\left.\mathbf{d}_{1}^{T} \mathbf{a}_{3} v_{x}-\mathbf{d}_{0}^{T} \mathbf{a}_{1} v_{x}\right)>0 .
\end{gathered}
$$

The constraints imposed by these two inequalities and the inequalities $F(0)<0$ arid $F(\Delta)<0$ provide a sufficient condition for error reduction.

\section{General Case}

The results presented in 2.1 and 2.2 can be generalized to the edge-vertex contact state involving both translational and orientational variations.

Suppose that at the four extremal configurations $\left([\delta, \theta]=\left[0, \theta_{m}\right],\left[0, \theta_{M}\right],\left[\Delta, \theta_{m}\right],\left[\Delta, \theta_{m}\right]\right)$ the error reduction condition and Condition $A$ are satisfied. Let $[\delta, \theta]$ be an arbitrary configuration with $\delta \in[0, \Delta]$ and $\theta \in\left[\theta_{m}, \theta_{M}\right]$.

Consider the two configurations determined by $\left[\delta, \theta_{m}\right]$ and $\left[\delta, \theta_{M}\right]$, by the results presented in Section 2.2, the error reduction condition must be satisfied at these two configurations. Then, by the results presented in Section 2.1, for any $\theta \in\left[\theta_{m}, \theta_{M}\right]$, the error reduction must be satisfied. Thus we have:

\section{Proposition 1:}

For an edge-vertex contact state, if at four configurations with different angle $\theta$ and contact locations $\delta$ the admittance satisfies Condition $A$ and the error reduction conditions, then the system will satisfy the error reduction conditions for all configurations bounded by these four configurations. 
Thus, for an edge-vertex contact state, to ensure the motion of error reduction of one body point, only four configuration extremals need be tested.

\section{SECTION III.}

\section{Vertex-edge Contact State}

In this section, the vertex-edge contact state is con-sidered. In this case, a vertex of the held body is in contact with an edge of the mating fixtured part (Fig. 1b). As shown, the configuration of the body can be determined by the orientation of the body $\theta$ and the location of the contact point $\delta$.

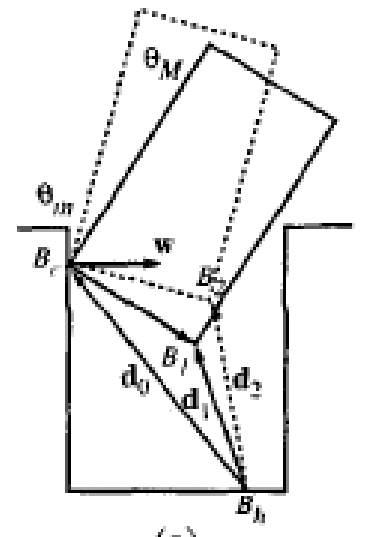

(a)

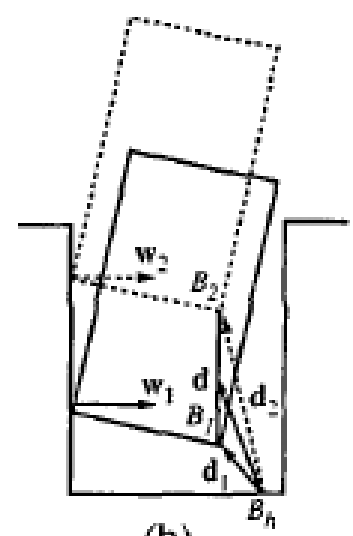

(b)

Fig, 3. Vertex-edge contact state. (a) orientational variation. (b) translational variation.

Suppose that $\theta$ varies within the range of $\left[\theta_{m}, \theta_{M}\right]$, and $\delta$ varies with $\mathbf{n}$ the range of $[0, \Delta]$. W e prove that for small variations of $\theta$ if an admittance matrix $A$ satisfies a set of conditions determined at the "bound-ary" configurations, then the system ensures that the motion is errorreducing for any intermediate configuration $\theta \in\left[\theta_{m}, \theta_{M}\right], \delta \in[0, \Delta]$. As in Section 2, we first consider orientational and translational variation separately. Finally, the general "vertex-edge" case is considered.

\section{A. Orientational Variation}

Consider only orientation variation as illustrated in Fig. 3a. In this case, both the direction of the error reduction vector $d$ and the direction of the contact force are changed by changing the orientation. We prove that, for small variation of $\theta$, if A satisfies a set of conditions at the 
orientation boundary $\theta m$ and $\theta M$, then an error-reducing motion is ensured for all configurations $\theta \in\left[\theta_{m}, \theta_{M}\right]$.

Let $\mathbf{w} 1$ and $\mathbf{w} 2$ be the two wrenches, and $\mathbf{d} 1$ and $\mathbf{d} 2$ be the two position vectors associated with $\theta \mathrm{m}$ and $\theta M$. Suppose at these two boundary points error-reducing motions are obtained, i.e.,

$$
\begin{aligned}
& \mathbf{d}_{1}{ }^{T} \mathbf{v}_{0}+\mathbf{d}_{1}{ }^{T} \mathbf{A} \mathbf{w}_{1} \leq 0 \\
& \mathbf{d}_{2}{ }^{T} \mathbf{v}_{0}+\mathbf{d}_{2}{ }^{T} \mathbf{A} \mathbf{w}_{2} \leq 0 .
\end{aligned}
$$

Consider a rotation given by an angle change $\theta$. The rotation matrix can be written as:

$$
\mathbf{R}(\theta)=\left[\begin{array}{cc}
\cos (\theta) & \sin (\theta) \\
-\sin (\theta) & \cos (\theta)
\end{array}\right]
$$

If we denote $\boldsymbol{n} m$ the surface normal associated with $\theta m$, then in the body coordination frame, the surface normal associated with $\theta$ is:

$$
\mathbf{n}_{\theta}=\mathbf{R n}_{m \cdot(23)}
$$

Since contact is frictionless, the contact force is along the surface normal at the contact point. Thus, the unit contact wrench is:

$$
\mathbf{w}_{n}(\theta)=\left[\begin{array}{c}
\mathbf{n}_{\theta} \\
\left(\mathbf{r} \times \mathbf{n}_{\theta}\right) \mathbf{k}
\end{array}\right]=\left[\begin{array}{c}
\mathbf{R} \mathbf{n}_{m} \\
\left(\mathbf{r} \times \mathbf{R} \mathbf{n}_{m}\right) \cdot \mathbf{k}
\end{array}\right]
$$

where $r$ is the position vector from the origin of the body frame to the contact point (constant) and $\mathrm{k}$ is the unit vector in the direction of the z-axis.

To determine the magnitude of the contact force associated with $\theta$, the reciprocal condition Eq. (9) is used, which yields

$$
\phi=-\frac{\mathbf{w}_{n}^{T} \mathbf{v}_{0}}{\mathbf{w}_{n}^{T} \mathbf{A} \mathbf{w}_{n}}(25)
$$

where $W n$ is the unit wrench defined in Eq. (24).

Since the two configurations correspond to pure rotation about the contact point, the position vector of $B$ for an intermediate configuration can be expressed in the body frame as:

$$
\mathbf{d}_{\theta}^{\prime}=\mathbf{R d}_{0}^{\prime}+\mathbf{d}^{\prime}(26)
$$


where $\mathbf{d}_{0}^{\prime}$ is the position vector from $B h$ to the contact point $B c, \mathbf{d}^{\prime}$ is the position vector from $B c$ to point $B 1$. Note that $\mathbf{d}_{0}^{\prime}$ is a constant in the global frame and $\mathbf{d}^{\prime}$ is constant in the body frame. Then, the line vector of $B$ relative to the body frame is obtained:

$$
\mathbf{d}_{\theta}=\left[\begin{array}{c}
\mathbf{d}_{\theta}^{\prime} \\
\left(\mathbf{r}_{\theta} \times \mathbf{d}_{\theta}^{\prime}\right) \cdot \mathbf{k}
\end{array}\right]
$$

Where $\mathbf{r}_{\theta}$ is the vector from the body frame origin to point $B$.

Therefore, the error reduction condition Eq. (2) can be expressed as a function of $\theta$ :

$$
\begin{gathered}
F(\theta)=\mathbf{d}_{\theta}^{T} \mathbf{v}_{0}+\mathbf{d}_{\theta}^{T} \mathbf{A} \mathbf{w}_{n} \phi \\
=\frac{\mathbf{d}_{\theta}^{T} \mathbf{v}_{0}\left(\mathbf{w}_{n}^{T} A \mathbf{w}_{n}\right)-\mathbf{d}_{\theta}^{T} A \mathbf{w}_{n}\left(\mathbf{w}_{n}^{T} \mathbf{v}_{0}\right)}{\mathbf{w}_{n}^{T} \mathbf{A} \mathbf{w}_{n}} .
\end{gathered}
$$

Since we only consider the sign of function $F(\theta)$ and $\mathbf{w}_{n}^{T} \mathbf{A} \mathbf{w}_{n}>0$, the error reduction function in Eq. (28) can be written as:

$$
G(\theta)=\mathbf{d}_{\theta}^{T} \mathbf{v}_{0}\left(\mathbf{w}_{n}^{T} \mathbf{A} \mathbf{w}_{n}\right)-\mathbf{d}_{\theta}^{\mathbf{T}} \mathbf{A} \mathbf{w}_{n}\left(\mathbf{w}_{n}^{T} \mathbf{v}_{0}\right) .
$$

It can be seen that $G(\theta)$ can be expressed analytically in terms of $\sin (\theta)$ and $\cos (\theta)$. If the variation of $\theta$ is small $(\leq 5 \circ), \sin (\theta)$ and $\cos (\theta)$ can be closely approximated by:

$$
\sin (\theta) \doteq \theta, \cos (\theta) \doteq 1-\frac{\theta^{2}}{2}
$$

With this approximation, the error reduction function $G(\theta)$ can be approximated by a 6 -th order polynomial having the form:

$$
G_{a}(\theta)=c_{6} \theta^{6}+c_{6} \theta^{\mathbf{s}}+\cdots+c_{2} \theta^{2}+c_{1} \theta+c_{0}
$$

"There each $c_{i}$ depends on the geometry parameter $\mathrm{d}$, and the admittance matrix $\mathrm{A}$.

Suppose that at the two configurations $\theta m$ and $\theta M$ we have error-reducing motions, i.e., $G_{a}\left(\theta_{m}\right)<0$ and $G_{a}\left(\theta_{M}\right)<0$. To ensure an error-reducing motion for an arbitrary configuration between $\theta m$ and $\theta_{M}, G_{a}(\theta)$ must be non-positive over the interval $\left[\theta_{m}, \theta_{M}\right]$ Math-ematically, the condition for error reduction implies that the polynomial $G_{a}(\theta)$ has no real root in $\left[\theta_{m}, \theta_{M}\right]$.

Consider the two sequences $Q_{1}$ and $Q_{2}$ defined by

$$
\begin{aligned}
& Q_{1}: G_{a}\left(\theta_{m}\right), G_{a}^{\prime}\left(\theta_{m}\right), \ldots, G_{a}^{(6)}\left(\theta_{m}\right) ; \\
& Q_{2}: G_{a}\left(\theta_{M}\right), G_{a}^{\prime}\left(\theta_{M}\right), \ldots, G_{a}^{(6)}\left(\theta_{M}\right) .
\end{aligned}
$$


Then, by the Fourier Theorem, $\mathrm{Ga}$ has no real root in $\left[\theta_{m}, \theta_{M}\right]$ if and only if the numbers of sign changes for $Q 1$ and $Q 2$ are the same. Since each element of $Q 1$ and $Q 2$ is a function of $A$, this condition can be stated as the following:

Condition $B$ : the admittance matrix $A$ satisfies the condition that the sequences $Q 1$ in Eq. (32) and $Q 2$ in Eq. (33) have the same number of sign changes.

Thus for small orientation variation, a sufficient condition for error-reducing motion is that 1 ) the admittance matrix A satisfies Condition $B$, and 2 ) at the two extremal angles the error reduction condition is satisfied.

\section{B. Translational Variation}

Now consider the translational variation of the contact configuration illustrated in Fig. 3b. In this case, only translation along the edge is allowed, and the contact force does not change in the body frame. The configuration of the body can be determined by a vector $d$ (Fig. 3b).

Suppose that, at the two locations $d_{1}$ and $d_{2}$, the error reduction conditions are satisfied:

$$
\begin{gathered}
\mathbf{d}_{1}{ }^{T} \mathbf{v}_{0}+\mathbf{d}_{1}{ }^{T} \mathbf{A} \mathbf{w}_{1} \leq 0 \\
\mathbf{d}_{2}{ }^{T} \mathbf{v}_{0}+\mathbf{d}_{2}{ }^{T} \mathbf{A} \mathbf{w}_{2} \leq 0
\end{gathered}
$$

where $\mathbf{w} 1$ and $\mathbf{w} 2$ are the contact wrenches at $\mathbf{d} 1$ and $\mathbf{d} 2$, respectively. Thus, for any $\alpha, \beta \geq 0$,

$$
\left(\alpha \mathbf{d}_{1}+\beta \mathbf{d}_{2}\right)^{T} \mathbf{v}_{0}+\left(\alpha \mathbf{d}_{1}+\beta \mathbf{d}_{2}\right)^{T} \mathbf{A w} \leq 0
$$

Consider an arbitrary configuration $\mathbf{d}$ between $\mathbf{d}_{1}$ and $\mathbf{d}_{2}$. Since the ends of these three vectors must be on a straight line, $\mathbf{d}$ is a convex combination of the vectors $\mathbf{d}_{1}$ and $\mathbf{d}_{2}$, i.e.,

$$
\mathbf{d}=\alpha \mathbf{d}_{1}+\beta \mathbf{d}_{2} \text { (37) }
$$

where $\alpha, \beta \geq 0$ and $\alpha+\beta=1$.

Since the contact wrench $w$ is the same in the body frame for all contact configurations, $\mathbf{w}=$ $\mathbf{w}_{1}=\mathbf{w}_{2}$. Substituting Eq. (37) into Eq. (36) yields:

$$
\mathbf{d}^{T} \mathbf{v}_{0}+\mathbf{d}^{T} \mathbf{A w} \leq 0
$$

Thus, for translational variation, if at two configurations the error reduction condition is satisfied, then the error reduction condition must be satisfied for all intermediate configurations bounded by these two con-figurations. 


\section{General Case}

Using the same reasoning presented in Section 2.3, the results in Sections 3.1 and 3.2 can be applied to the vertex-edge contact case involving both translational and orientational variations.

\section{Proposition 2:}

For a vertex-edge contact state with small variation of orientation $\left[\theta_{m}, \theta_{M}\right]$ and finite variation of translation, if at the four boundary configurations the error reduction conditions and Condition $B$ are satisfied, then the error reduction condition is satisfied for all intermediate configurations bounded by these four configurations.

Thus, for an edge-vertex contact state, to ensure the motion of error reduction of one body point, only four configuration extremals need be tested.

\section{SECTION IV.}

\section{Discussion}

In this paper, the error reduction condition for a single point on the held body is considered. Since this point is arbitrary, the results can be extended to a set of finite points. For example, if $n$ points on the body are considered, the conditions in Propositions 1 and 2 must be satisfied for all of the $n$ points.

Condition A and Condition B are for the vertex-edge and edge-vertex contact states respectively. Both conditions can be formulated as a set of inequalities. It should be noted that Condition $B$ is valid only for small orientation variation. If the orientation variation is significant, a higher order polynomial should be used in Eq. (30). As a consequence, the order of the polynomial Ga in Eq. (31) and the number of terms in sequences (32) and (33) are increased. The conditions provided in the paper can be used as constraints in searching for an appropriate admittance A.

\section{SECTION V.}

\section{Summary}

In this paper, admittance selection of a planar rigid body motion for force-guided assembly is addressed. We have shown that, for one point contact cases, the admittance control law can be selected based on their behavior on a finite number of configurations. If the error reduction conditions are satisfied at these config-urations, the error reductions will be satisfied for all intermediate configurations. Thus, for a given set of bounded misalignments, a single admittance control law can guarantee the proper assembly of a given pair of mating parts. 


\section{ACKNOWLEDGMENTS}

This research was supported in part by a Ford Motor Company URP award and the National

Science Foundation under grant IIS 0010017.

\section{References}

1. D. E. Whitney, "Force feedback control of manipulator fine motions", ASME Journal of Dynamic Systems: Measurements and Control, vol. 98, no. 2, 1977.

2. D. E. Whitney, "Quasi-static assembly of compliantly supported rigid parts", ASME Journal of Dynamic Systems Measurements and Control, vol. 104, no. 1, pp. 65-77, 1982.

3. M. A. Peshkin, "Programmed compiance for error-corrective manipulation", IEEE Transactions on Robotics and Automation, vol. 6, no. 4, pp. 473-482, 1990.

4. H. Asada, "Teaching and learning of compliance using neural net", IEEE Transactions on Robotics and Automation, vol. 9, no. 6, pp. 863-867, 1993.

5. E. D. Fasse, Jan F. Broenink, "A spatial impedance controller for robotic manipulation", IEEE Transactions on Robotics and Automation, vol. 13, no. 4, 1997.

6. H. Ang Marcelo, Gerry B. Andeen, "Specifying and achieving passive compliance based on manipulator structure", IEEE Transactions on Robotics and Automation, vol. 11, no. 4., 1995.

7. J. M. Schimrnels, M. A Peshkin, "Admittance matrix design for force guided assembly", IEEE Transactions on Robotics and Automation; vol. 8, no. 2, pp. 213-227, 1992.

8. J. M Schimrnels, M A. Peshkin, "Force-assembly with friction", IEEE Transactions on Robotics and Automation, vol. 10, no. 4, pp. 465-479, 1994.

9. Joseph M. Schimrnels, "A linear space of admittance control laws that guarantees forceassembly with friction", IEEE Transactions on Robotics and Automation, vol. 13, no. 5, pp. 656-667, 1997.

10. J. M R. Martinez, J. Duffy, "On the metric of rigid body displacements for infinite and finite bodies", ASME Journal of Mechanical Design, vol. 117, no. 1, 1995. 